Document downloaded from:

http://hdl.handle.net/10251/67201

This paper must be cited as:

Bivià-Ausina, C. (2015). Multiplicity and Lojasiewicz exponent of generic linear sections of monomial ideals. Bulletin of the Australian Mathematical Society. 91(2):191-201. doi:10.1017/S0004972714001154.

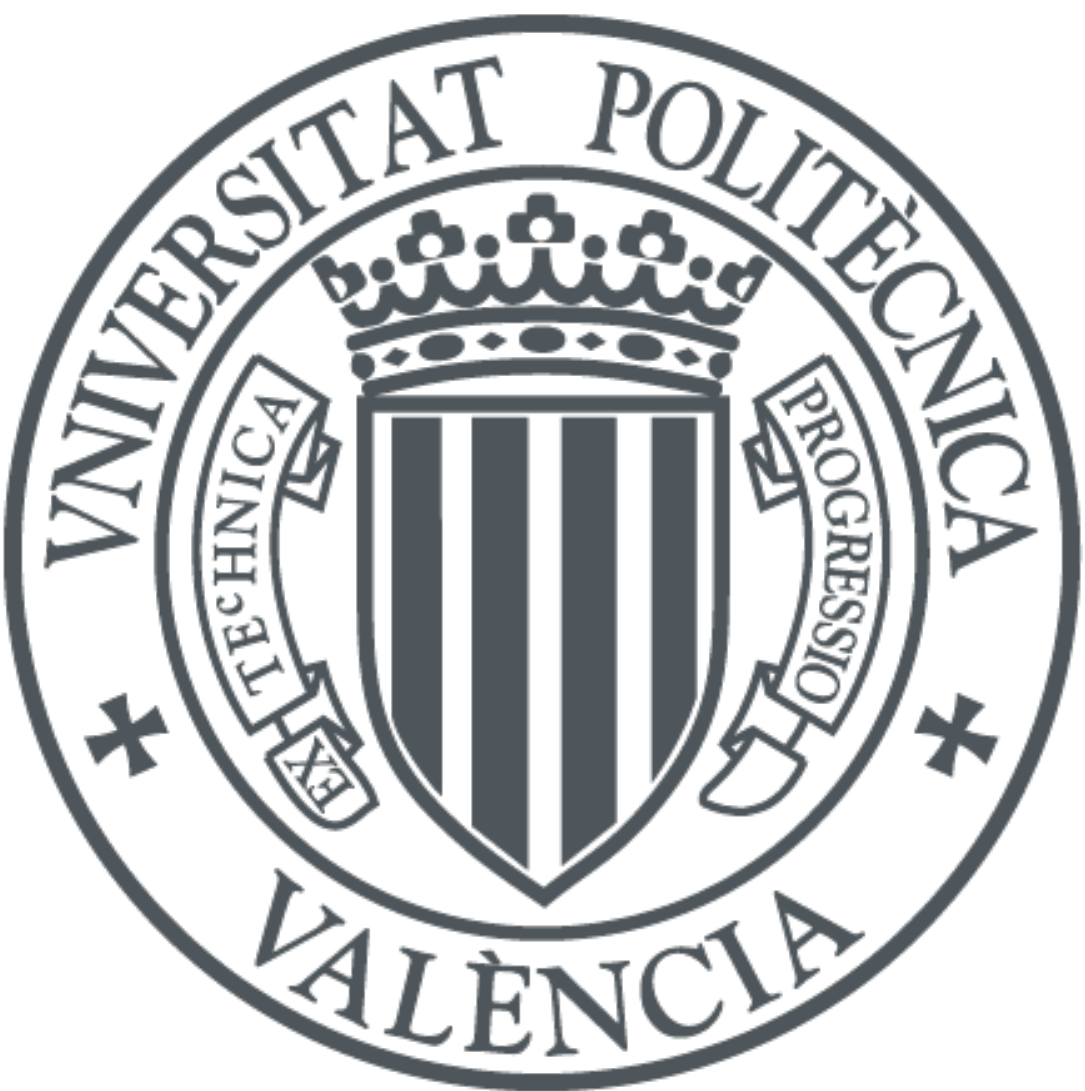

The final publication is available at

http://dx.doi.org/10.1017/S0004972714001154

Copyright Cambridge University Press (CUP) + Australian Mathematical Publishing Association Inc.

Additional Information 


\title{
MULTIPLICITY AND ŁOJASIEWICZ EXPONENT OF GENERIC LINEAR SECTIONS OF MONOMIAL IDEALS
}

\author{
C. BIVIÀ-AUSINA
}

\begin{abstract}
We obtain a characterization of the monomial ideals $I \subseteq \mathbb{C}\left[x_{1}, \ldots, x_{n}\right]$ of finite colength that satisfy the condition $e(I)=\mathcal{L}_{0}^{(1)}(I) \cdots \mathcal{L}_{0}^{(n)}(I)$, where $\mathcal{L}_{0}^{(1)}(I), \ldots, \mathcal{L}_{0}^{(n)}(I)$ is the sequence of mixed Łojasiewicz exponents of $I$ and $e(I)$ is the Samuel multiplicity of $I$. These are the monomial ideals whose integral closure admits a reduction generated by homogeneous polynomials.
\end{abstract}

\section{INTRODUCTION}

Let $(R, \mathbf{m})$ denote a local ring of dimension $n$. Let $I$ be an $\mathbf{m}$-primary ideal of $R$. There are two important numbers attached to $I$ : the multiplicity of $I$, denoted by $e(I)$ (see for instance [9], [14] or [24]), and the Łojasiewicz exponent of $I$, that is usually denoted by $\mathcal{L}_{0}(I)$ (see [15], [22] and [23]). We shall also refer to $\mathbf{m}$-primary ideals as ideals of finite colength. We recall that $\mathcal{L}_{0}(I)$ is originally defined for ideals of the ring $\mathcal{O}_{n}$ of analytic function germs $\left(\mathbb{C}^{n}, 0\right) \rightarrow \mathbb{C}$ around the origin. That is if $I$ is generated by $g_{1}, \ldots, g_{r} \in \mathcal{O}_{n}$, then $\mathcal{L}_{0}(I)$ is defined as the infimum between all positive real numbers $\alpha$ such that

$$
\|x\|^{\alpha} \leqslant C \sup _{i}\left|g_{i}(x)\right|
$$

for some constant $C>0$ and all $x$ that belongs to some open neighbourhood of the origin in $\mathbb{C}^{n}$. Lejeune and Teissier showed in [15] a relation between $\mathcal{L}_{0}(I)$ and the asymptotic Samuel function of $I$ and, consequently, with the integral closure of $I$. This relation is the motivation of the definition of $\mathcal{L}_{0}(I)$ for an arbitrary ideal $I$ of finite colength in a local ring $(R, \mathbf{m})$. Let us explain this more precisely.

Let us fix a local ring $(R, \mathbf{m})$. Let $I$ be an ideal of $R$ and let $h \in R$. Then the order of $h$

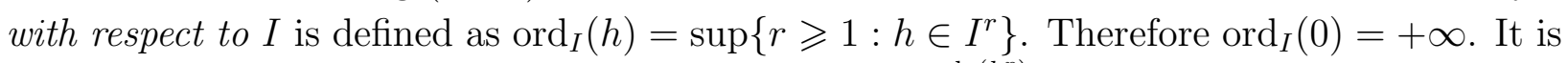
proven in [15, Section 0.2] and [17] that the sequence $\left\{\frac{\operatorname{ord}_{I}\left(h^{r}\right)}{r}\right\}_{r \geqslant 1}$ has a limit in $\mathbb{R}_{\geqslant 0} \cup\{+\infty\}$. The asymptotic Samuel function of $I$ is defined as the function $\bar{\nu}_{I}: R \rightarrow \mathbb{R}_{\geqslant 0} \cup\{+\infty\}$ given by

$$
\bar{\nu}_{I}(h)=\lim _{r \rightarrow \infty} \frac{\operatorname{ord}_{I}\left(h^{r}\right)}{r}
$$

2010 Mathematics Subject Classification. Primary 13B22; Secondary 13H15.

Key words and phrases. Lojasiewicz exponents, integral closure of ideals, mixed multiplicities of ideals, monomial ideals, Newton polyhedra.

The first author was partially supported by DGICYT Grant MTM2012-33073. 
for all $h \in R$, where we set $\bar{\nu}_{I}(0)=+\infty$. We remark that $\bar{\nu}_{I}(h)=0$, for all $h \notin \sqrt{I}$. The number $\bar{\nu}_{I}(h)$ is also known as the reduced order of $h$ with respect to $I$. It is known that the range of $\bar{\nu}_{I}$ is $\mathbb{Q}_{\geqslant 0} \cup\{+\infty\}$ (see for instance [14, $\left.\S 10\right]$ ).

If $I$ is an ideal of $\mathcal{O}_{n}$ such that $\bar{I}$ is a monomial ideal (that is, $\bar{I}$ is generated by monomials) then $\bar{\nu}_{I}$ can be expressed in terms of the Newton polyhedron of $I$ (see [2]). If $I$ and $J$ are ideals of $R$, then we define

$$
\bar{\nu}_{I}(J)=\min \left\{\bar{\nu}_{I}(h): h \in J\right\} .
$$

Thus the result of Lejeune-Teissier to which we referred before says that, if $I$ is an ideal of $\mathcal{O}_{n}$ of finite colength and $\mathbf{m}_{n}$ denotes the maximal ideal of $\mathcal{O}_{n}$, then

$$
\mathcal{L}_{0}(I)=\frac{1}{\bar{\nu}_{I}\left(\mathbf{m}_{n}\right)} .
$$

The above equality is used as the definition of Łojasiewicz exponent of an arbitrary ideal $I$ of finite colength in a local ring $(R, \mathbf{m})$. We also remark that equality $(1)$ is equivalent to $\mathcal{L}_{0}(I)=\inf \left\{\frac{r}{s}: r, s \in \mathbb{Z}_{\geqslant 1}, \mathbf{m}_{n}^{r} \subseteq \overline{I^{s}}\right\}$ (see [15, Section 7$]$ ).

The notion of multiplicity of an ideal was extended by Risler and Teissier [22] to sequences of $\mathbf{m}$-primary ideals thus leading to the notion of mixed multiplicity $e\left(I_{1}, \ldots, I_{n}\right)$ of $n \mathbf{m}$ primary ideals in $R$ (see $[14, \S 17.4]$ ). The motivation of this generalization has its origin in the study developed by Teissier of the Milnor number of the restriction of a given function germ $f \in \mathcal{O}_{n}$ to generic subspaces of $\mathbb{C}^{n}$ of different dimensions [22]. The study of mixed multiplicities of ideals was further developed by Rees in [19]. Let $(R, m)$ be a local ring and let $I_{1}, \ldots, I_{n}$ be ideals of $R$. Then we define

$$
\sigma\left(I_{1}, \ldots, I_{n}\right)=\sup _{r \in \mathbb{Z} \geqslant 1} e\left(I_{1}+\mathbf{m}^{r}, \ldots, I_{n}+\mathbf{m}^{r}\right) .
$$

In general $\sigma\left(I_{1}, \ldots, I_{n}\right)$ can be infinite. In [4, p. 393] we characterized the finiteness of $\sigma\left(I_{1}, \ldots, I_{n}\right)$. From $(2)$ it is clear that if each ideal has finite colength then $\sigma\left(I_{1}, \ldots, I_{n}\right)$ exists and it is equal to the usual mixed multiplicity $e\left(I_{1}, \ldots, I_{n}\right)$. We remark that $\sigma\left(I_{1}, \ldots, I_{n}\right)$ coincides with the mixed multiplicity defined by Rees in [18, p. 181]. Then we also refer to $\sigma\left(I_{1}, \ldots, I_{n}\right)$ as the Rees' mixed multiplicity of $I_{1}, \ldots, I_{n}$.

Analogous to the generalization of the notion of multiplicity leading to mixed multiplicities, we introduced in [3] the notion of Łojasiewicz exponent of $n$ ideals $I_{1}, \ldots, I_{n}$ in a local ring $(R, \mathbf{m})$ of dimension $n$ (see Section 2 and [5] for details). We denote this number by $\mathcal{L}_{0}\left(I_{1}, \ldots I_{n}\right)$. In order to define $\mathcal{L}_{0}\left(I_{1}, \ldots I_{n}\right)$, the ideals $I_{1}, \ldots, I_{n}$ are not assumed to have finite colength but the condition $\sigma\left(I_{1}, \ldots, I_{n}\right)<\infty$ is needed. Therefore, if $I$ denotes an ideal of finite colength of $R$ and $i \in\{1, \ldots, n\}$, then we define the $i$-th relative Eojasiewicz exponent of I as

$$
\mathcal{L}_{0}^{(i)}(I)=\mathcal{L}_{0}(I, \ldots, I, \mathbf{m}, \ldots, \mathbf{m}),
$$

where $I$ is repeated $i$ times and $m$ is repeated $n-i$ times. In particular we have $\mathcal{L}_{0}^{(n)}(I)=$ $\mathcal{L}_{0}(I)$ and $\mathcal{L}_{0}^{(1)}(I)=\operatorname{ord}(I)$. 
Let $(R, \mathbf{m})$ denote an equicharacteristic regular local ring of dimension $n \geqslant 2$ with residue field $\mathbf{k}, \operatorname{char}(\mathbf{k})=0$. Let $I$ be an ideal of $R$ of finite colength and let us fix an index $i \in\{1, \ldots, n\}$. M. Hickel proved in [12, Théorème 1.1] that there exists a Zariski open set $U^{(i)}$ of the Grassmannian $G_{\mathbf{k}}(i, n)$ of subspaces of dimension $i$ of $\mathbf{k}^{n}$ such that $\bar{\nu}_{I R_{H}}\left(\mathbf{m}_{H}\right)$ does not depend on $H$, for all $H \in U^{(i)}$, where we assume that $H$ is the zero set of the collection of k-linear forms $h_{1}, \ldots, h_{n-i}$, the quotient ring $R /\left\langle h_{1}, \ldots, h_{n-i}\right\rangle$ is denoted by $R_{H}$ and $\mathbf{m}_{H}$ is the maximal ideal of $R_{H}$. By [6, Lemma 4.9], we have $\mathcal{L}_{0}^{(i)}(I)=\left(\bar{\nu}_{I R_{H}}\left(\mathbf{m}_{H}\right)\right)^{-1}$, for all $i=1, \ldots, n$. We remark that $\left(\bar{\nu}_{I R_{H}}\left(\mathbf{m}_{H}\right)\right)^{-1}$ is denoted by $\nu_{I}^{(i)}$ in [12], for all $i=1, \ldots, n$.

Moreover Hickel proved in [12] that

$$
e(I) \leqslant \mathcal{L}_{0}^{(1)}(I) \cdots \mathcal{L}_{0}^{(n)}(I)
$$

We remark that this inequality was generalized in [6, Theorem 4.7]. Then there appears the problem of characterizing when equality holds in (3) and to understand the structure of the ideals satisfying that equality. This is already done by Hickel in dimension $n=2$ in [12, Proposition 5.1].

In this article we consider this problem when we suppose that $I$ is a monomial ideal of $\mathcal{O}_{n}$ or $\mathbb{C}\left[\left[x_{1}, \ldots, x_{n}\right]\right]$ (see Theorem 3.5). We prove that the only monomial ideals that satisfy the equality $e(I)=\mathcal{L}_{0}^{(1)}(I) \cdots \mathcal{L}_{0}^{(n)}(I)$ are those such that $\bar{I}$ admits a reduction generated by homogeneous polynomials. This conditions reduces considerably the possibilities for the shape of the Newton polyhedron of the ideal. As is seen in Section 3, we translate this problem into a combinatorial problem, that, at first sight is independent from Łojasiewicz exponents and captures a special class of monomial ideals.

\section{Mixed ŁoJASIEWiCZ EXPONEnts}

In this section we recall briefly the notion of mixed Łojasiewicz exponent and some basic facts about this concept.

Let $(R, \mathbf{m})$ denote a Noetherian local ring of dimension $n \geqslant 1$ and let $I_{1}, \ldots, I_{n}$ be ideals of $R$ such that $\sigma\left(I_{1}, \ldots, I_{n}\right)<\infty$. Let $J$ be a proper ideal of $R$. Let us define

$$
r_{J}\left(I_{1}, \ldots, I_{n}\right)=\min \left\{r \in \mathbb{Z}_{\geqslant 0}: \sigma\left(I_{1}, \ldots, I_{n}\right)=\sigma\left(I_{1}+J^{r}, \ldots, I_{n}+J^{r}\right)\right\} .
$$

We recall that $\sigma\left(I_{1}, \ldots, I_{n}\right)$ denotes the Rees' mixed multiplicity of $I_{1}, \ldots, I_{n}$, defined in (2).

If we suppose that $I_{1}=\cdots=I_{n}=I$, for some ideal $I$ of $R$ of finite colength and we assume that $R$ is formally equidimensional, then we can apply the Rees's multiplicity theorem (see $[11$, p. 147$]$ or $[14$, p. 222]) to deduce that

$$
r_{J}(I, \ldots, I)=\min \left\{r \in \mathbb{Z}_{\geqslant 0}: J^{r} \subseteq \bar{I}\right\} .
$$

Definition 2.1. [5] Under the above conditions, we define the Eojasiewicz exponent of $I_{1}, \ldots, I_{n}$ with respect to $J$, denoted by $\mathcal{L}_{J}\left(I_{1}, \ldots, I_{n}\right)$, as

$$
\mathcal{L}_{J}\left(I_{1}, \ldots, I_{n}\right)=\inf _{s \geqslant 1} \frac{r_{J}\left(I_{1}^{s}, \ldots, I_{n}^{s}\right)}{s} .
$$


We also refer to the number $\mathcal{L}_{J}\left(I_{1}, \ldots, I_{n}\right)$ as the mixed Eojasiewicz exponent of $I_{1}, \ldots, I_{n}$ with respect to $J$. When $J=\mathbf{m}$ then we denote this number by $\mathcal{L}_{0}\left(I_{1}, \ldots, I_{n}\right)$.

Let us observe that in order to define $\mathcal{L}_{J}\left(I_{1}, \ldots, I_{n}\right)$ we only need the ring $R$ to be local and Noetherian, no additional condition on $R$ is assumed. As mentioned in the Introduction, if $I$ is an ideal of finite colength of $R$, then we can associate to $I$ the vector $\mathcal{L}_{0}^{*}(I)=$ $\left(\mathcal{L}_{0}^{(n)}(I), \ldots, \mathcal{L}_{0}^{(1)}(I)\right)$, where $\mathcal{L}_{0}^{(i)}(I)=\mathcal{L}_{0}(I, \ldots, I, \mathbf{m}, \ldots, \mathbf{m})$, with $I$ repeated $i$ times and m repeated $n-i$ times, $i=1, \ldots, n$. The number $\mathcal{L}_{J}^{(i)}(I)$ is defined analogously, for all $i=1, \ldots, n$, and any ideal $J$ of $R$ of finite colength.

The following result is proven in [6, Corollary 4.11].

Theorem 2.2. Let $(R, \mathbf{m})$ be a quasi-unmixed Noetherian local ring and let $I, J$ be ideals of $R$ of finite colength. Let us suppose that the residue field $k=R / \mathbf{m}$ is infinite. Then $\mathcal{L}_{J}^{(1)}(I) \leqslant \cdots \leqslant \mathcal{L}_{J}^{(n)}(I)$.

Let us fix coordinates $x_{1}, \ldots, x_{n}$ in $\mathbb{C}^{n}$. If $k \in \mathbb{Z}_{\geqslant 0}^{n}$, then we write $x^{k}$ to denote the monomial $x_{1}^{k_{1}} \cdots x_{n}^{k_{n}}$. If $h \in \mathcal{O}_{n}$ and the Taylor expansion of $h$ around the origin is given by $h=\sum_{k} a_{k} x^{k}$, then the support of $h$, denoted by $\operatorname{supp}(h)$, is defined as the set of those $k \in \mathbb{Z}_{\geqslant 0}^{n}$ such that $a_{k} \neq 0$. If $h \neq 0$, then we define the Newton polyhedron of $h$, denoted by $\Gamma_{+}(h)$, as the convex hull in $\mathbb{R}_{\geqslant 0}^{n}$ of $\left\{k+v: k \in \operatorname{supp}(h), v \in \mathbb{R}_{+}^{n}\right\}$. If $h=0$, then we set $\Gamma_{+}(h)=\emptyset$. If $I$ is an ideal of $\mathcal{O}_{n}$, then the Newton polyhedron of $I$, denoted by $\Gamma_{+}(I)$, is defined as the convex hull of $\Gamma_{+}\left(g_{1}\right) \cup \cdots \cup \Gamma_{+}\left(g_{s}\right)$, where we assume that $g_{1}, \ldots, g_{s}$ is a generating system of $I$. It is immediate to see that the definition of $\Gamma_{+}(I)$ does not depend on the chosen generating system of $I$.

Let us fix a subset $\mathrm{L} \subseteq\{1, \ldots, n\}, \mathrm{L} \neq \emptyset$. Then we denote by $\mathbb{R}_{\mathrm{L}}^{n}$ the set of those $k \in \mathbb{R}^{n}$ such that $k_{j}=0$, for all $j \notin \mathrm{L}$. If $A$ denotes any subset of $\mathbb{R}^{n}$ then we set $A^{\mathrm{L}}=A \cap \mathbb{R}_{\mathrm{L}}^{n}$. The cardinal of $\mathrm{L}$ will be denoted by $|\mathrm{L}|$.

If $I$ is a monomial ideal of $\mathcal{O}_{n}$, then we denote by $I^{\mathrm{L}}$ the ideal of $\mathcal{O}_{n}$ generated by the monomials $x^{k} \in I$ such that $k \in \mathbb{R}_{\mathrm{L}}^{n}$. If $\operatorname{supp}(I) \cap \mathbb{R}_{\mathrm{L}}^{n}=\emptyset$, then we set $I^{\mathrm{L}}=0$. If $I$ is a monomial ideal of $\mathcal{O}_{n}$ of finite colength, then we have $I^{\mathrm{L}} \neq 0$, for all $\mathrm{L} \subseteq\{1, \ldots, n\}, \mathrm{L} \neq \emptyset$.

The next result gives a description of the sequence $\mathcal{L}_{0}^{*}(I)$ in terms of $\Gamma_{+}(I)$ when $I$ is a monomial ideal of finite colength of $\mathcal{O}_{n}$.

Theorem 2.3. [6] Let $I$ be a monomial ideal of $\mathcal{O}_{n}$ of finite colength. Let $i \in\{1, \ldots, n\}$. Then

$$
\mathcal{L}_{0}^{(i)}(I)=\max \left\{\operatorname{ord}\left(I^{\mathrm{L}}\right): \mathrm{L} \subseteq\{1, \ldots, n\},|\mathrm{L}|=n-i+1\right\}
$$

The following result is motivated by [12, Théorème 1.1] and in turn, the case $J=\mathbf{m}$ is the motivation of the problem considered in this article. This result can be seen as a particular case of [6, Theorem 4.7] (see [6, Corollary 4.8]). We also refer to [12, Remarque 4.3 (3)] for the deduction of inequality (6) using different techniques in a slightly different context. 
Proposition 2.4. [6] Let $(R, \mathbf{m})$ be a quasi-unmixed Noetherian local ring and let $I$ and $J$ be ideals of $R$ of finite colength. Then

$$
\frac{e(I)}{e(J)} \leqslant \mathcal{L}_{J}^{(1)}(I) \cdots \mathcal{L}_{J}^{(n)}(I)
$$

In the main result, Theorem 3.5, we obtain a characterization of when equality holds in (6) considering that $I$ is a monomial ideal of $\mathcal{O}_{n}$ and $J$ is the maximal ideal of $\mathcal{O}_{n}$. As we will see, Theorem 3.5 can be considered as a purely combinatorial result.

\section{MAIN RESULT}

In this section we expose the concepts and results from [21] that we need in the proof of the main result.

If $A$ is a subset of $\mathbb{R}^{n}$, then we denote by $\operatorname{Conv}(A)$ the convex hull of $A$ in $\mathbb{R}^{n}$. If $P \subseteq \mathbb{R}^{n}$, then we say that $P$ is a polytope when there exists a finite subset $A \subseteq \mathbb{R}^{n}$ such that $P=\operatorname{Conv}(A)$. If $A$ is contained in $\mathbb{Z}^{n}$, then $\operatorname{Conv}(A)$ is said to be lattice polytope.

If $P$ is a polytope in $\mathbb{R}^{n}$, then the dimension of $P$ is defined as the minimum dimension of an affine subspace containing $P$.

If $P$ is any subset of $\mathbb{R}^{n}$, then we denote by $\mathbb{C}[P]$ the family of polynomial maps $h \in$ $\mathbb{C}\left[x_{1}, \ldots, x_{n}\right]$ such that $\operatorname{supp}(h) \subseteq P$. Let $\mathbf{P}=\left(P_{1}, \ldots, P_{n}\right)$ be an $n$-tuple of subsets of $\mathbb{R}^{n}$. We denote by $\mathbb{C}_{n}[\mathbf{P}]$ the set of polynomial maps $F=\left(F_{1}, \ldots, F_{n}\right): \mathbb{C}^{n} \rightarrow \mathbb{C}^{n}$ such that $\operatorname{supp}\left(F_{i}\right) \subseteq P_{i}$, for all $i=1, \ldots, n$. We can identify $\mathbb{C}_{n}[\mathbf{P}]$ with a finite dimensional vector space $\mathbb{C}^{N}$, for a big enough positive integer $N$, by associating to each map $F \in \mathbb{C}_{n}[\mathbf{P}]$ the vector formed by the coefficients of $F$. Under this identification, we say that a property holds for a generic $F \in \mathbb{C}_{n}[\mathbf{P}]$ when the said property holds in a dense Zariski open subset of $\mathbb{C}^{N}$.

Given a lattice polytope $P \subseteq \mathbb{R}^{n}$, then we say that $P$ is cornered when for all $j=1, \ldots, n$ there exists some $k \in P$ such that $k_{j}=0$ (see [21, p. 119]). If $P \subseteq \mathbb{R}_{\geqslant 0}^{n}$ and $f_{P}$ denotes the polynomial obtained as the sum of all terms $x^{k}$ such that $k \in P \cap \mathbb{Z}_{\geqslant 0}^{n}$, then we observe that $P$ is cornered if and only if $f_{P}$ is not divisible by $x_{j}$, for all $j=1, \ldots, n$.

Let $\mathbf{P}=\left(P_{1}, \ldots, P_{n}\right)$ be an $n$-tuple of lattice polytopes in $\mathbb{R}^{n}$, then $\mathbf{P}$ is said to be cornered when $P_{i}$ is cornered, for all $i=1, \ldots, n$. We say that $\mathbf{P}$ is nice when $F^{-1}(0)$ is finite, for a generic map $F \in \mathbb{C}_{n}[\mathbf{P}]$.

If $F: \mathbb{C}^{n} \rightarrow \mathbb{C}^{n}$ is a polynomial map such that $F^{-1}(0)$ is finite, then we denote by $m(F)$ the number of roots of $F$ counted with multiplicities. That is, if $I(F)$ denotes the ideal of $\mathbb{C}\left[x_{1}, \ldots, x_{n}\right]$ generated by the component functions of $F$, then applying [7, Chapter 4 , Corollary 2.5] we have

$$
m(F)=\operatorname{dim}_{\mathbb{C}} \frac{\mathbb{C}\left[x_{1}, \ldots, x_{n}\right]}{I(F)} .
$$

If $K \subseteq \mathbb{R}^{n}$, then we denote by $\operatorname{Vol}_{n}(K)$ the $n$-dimensional volume of $K$. Let $C_{1}, \ldots, C_{n}$ be $n$ polytopes of $\mathbb{R}^{n}$ and let $\lambda_{1}, \ldots, \lambda_{n} \in \mathbb{R}_{\geqslant 0}$. Let $\lambda_{1} C_{1}+\cdots+\lambda_{n} C_{n}=\left\{\lambda_{1} k_{1}+\cdots+\lambda_{n} k_{n}: k_{i} \in\right.$ 
$\left.C_{i}, i=1, \ldots, n\right\}$. It is a classical result from convex geometry that $\operatorname{Vol}_{n}\left(\lambda_{1} C_{1}+\cdots+\lambda_{n} C_{n}\right)$ is a homogeneous polynomial of degree $n$ in the variables $\lambda_{1}, \ldots, \lambda_{n}$ (see for instance [7, p. 337]). The $n$-dimensional mixed volume of $C_{1}, \ldots, C_{n}$ is defined as the coefficient of $\lambda_{1} \cdots \lambda_{n}$ in the polynomial $\operatorname{Vol}_{n}\left(\lambda_{1} C_{1}+\cdots+\lambda_{n} C_{n}\right)$. We denote this number by $M\left(C_{1}, \ldots, C_{n}\right)$. Let us recall some elementary properties of this number (we take them from [20, p. 112], see also [7, Chapter $7, \S 4])$ :

(1) $M\left(C_{1}, \ldots, C_{n}\right)$ is symmetric and linear in each variable.

(2) $M\left(C_{1}, \ldots, C_{n}\right) \geqslant 0$ and $M\left(C_{1}, \ldots, C_{n}\right)=0$ if and only if $\operatorname{dim}\left(\sum_{i \in I} C_{i}\right)<|I|$, for some non-empty subset $I \subseteq\{1, \ldots, n\}$, where $|I|$ denotes the cardinal of $I$.

(3) $M\left(C_{1}, \ldots, C_{n}\right) \in \mathbb{Z}_{\geqslant 0}$, if $C_{i}$ is a lattice polytope, for all $i=1, \ldots, n$.

(4) $M(C, \ldots, C)=n ! \operatorname{Vol}_{n}(C)$, for any polytope $C \subseteq \mathbb{R}^{n}$.

We refer to $[7,10,20]$ for more information about $M\left(C_{1}, \ldots, C_{n}\right)$.

If $P$ is a polytope in $\mathbb{R}^{n}$, then we denote $\operatorname{Conv}(P \cup\{0\})$ by $P^{0}$. If $\mathbf{P}=\left(P_{1}, \ldots, P_{n}\right)$ is an $n$-tuple of polytopes of $\mathbb{R}^{n}$, then we define $\mathbf{P}^{0}=\left(P_{1}^{0}, \ldots, P_{n}^{0}\right)$. In particular, it makes sense to speak about the mixed volumes $M(\mathbf{P})$ and $M\left(\mathbf{P}^{0}\right)$. Let us remark that $\mathbf{P}^{0}$ is always cornered. By [16, Theorem 2.4], if $F \in \mathbb{C}_{n}[\mathbf{P}], \mathbf{P}$ is a lattice polytope and $F^{-1}(0)$ is finite, then $m(F) \leqslant M\left(\mathbf{P}^{0}\right)$. As remarked in [21, p. 119], the conditions nice and cornered on $\mathbf{P}$ are independent conditions. The following results tells us that both properties together in $\mathbf{P}$ imply $m(F)=M\left(\mathbf{P}^{0}\right)$, for a generic $F \in \mathbb{C}_{n}[\mathbf{P}]$.

Theorem 3.1. [21, p. 119] Let $\mathbf{P}=\left(P_{1}, \ldots, P_{n}\right)$ be an $n$-tuple of lattice polytopes of $\mathbb{R}_{\geqslant 0}^{n}$. Let us suppose that $\mathbf{P}$ is nice and cornered. Then a generic polynomial map $F \in \mathbb{C}_{n}[\mathbf{P}]$ has exactly $M\left(\mathbf{P}^{0}\right)$ roots in $\mathbb{C}^{n}$, counting multiplicities.

The previous theorem is proven in [21] in a more general context (i.e. for polynomial maps with coefficients in a given algebraically closed field of any characteristic).

Let $\mathbf{P}=\left(P_{1}, \ldots, P_{n}\right)$ and $\mathbf{Q}=\left(Q_{1}, \ldots, Q_{n}\right)$ be $n$-tuples of polytopes in $\mathbb{R}_{+}^{n}$. We write $\mathbf{P} \subseteq \mathbf{Q}$ to denote that $P_{i} \subseteq Q_{i}$, for all $i=1, \ldots, n$. We also define the $n$-tuples of subsets $\mathbf{P} \cap \mathbf{Q}=\left(P_{1} \cap Q_{1}, \ldots, P_{n} \cap Q_{n}\right)$ and $\mathbf{Q} \backslash \mathbf{P}=\left(Q_{1} \backslash P_{1}, \ldots, Q_{n} \backslash P_{n}\right)$. If $\mathrm{L} \subseteq\{1, \ldots, n\}$, $\mathrm{L} \neq \emptyset$, then we set $\mathbf{P}^{\mathrm{L}}=\left(P_{1} \cap \mathbb{R}_{\mathrm{L}}^{n}, \ldots, P_{n} \cap \mathbb{R}_{\mathrm{L}}^{n}\right)$.

Next we recall a particular case of a definition introduced in [21, p. 120].

Definition 3.2. Let $\mathbf{P}$ and $\mathbf{Q}$ be $n$-tuples of polytopes in $\mathbb{R}_{\geqslant 0}^{n}$ such that $\mathbf{Q}$ is nice and cornered. We say that $\mathbf{P}$ counts $\mathbf{Q}$ when

(1) $\mathbf{P} \subseteq \mathbf{Q}$

(2) $\mathbf{P}$ is nice

(3) For any $F \in \mathbb{C}_{n}[\mathbf{Q} \backslash \mathbf{P}]$, the map $F+F^{\prime}$ has a finite zero set and $m\left(F+F^{\prime}\right)=M\left(\mathbf{Q}^{0}\right)$, for a generic $F^{\prime} \in \mathbb{C}_{n}[\mathbf{P}]$.

In particular, if $\mathbf{P}$ counts $\mathbf{Q}$, then $m(F)=M\left(\mathbf{Q}^{0}\right)$, for a generic $F \in \mathbb{C}_{n}[\mathbf{P}]$, and therefore $M\left(\mathbf{P}^{0}\right)=M\left(\mathbf{Q}^{0}\right)$ provided that $\mathbf{P}$ is also cornered, by Theorem 3.1. 
Definition 3.3. [21, p. 124] Let $\mathbf{P}=\left(P_{1}, \ldots, P_{n}\right)$ be an $n$-tuple of polytopes in $\mathbb{R}^{n}$. The support of $\mathbf{P}$ is defined as the set of indices $i \in\{1, \ldots, n\}$ such that $P_{i} \neq \emptyset$. We denote this set by $\operatorname{supp}(\mathbf{P})$. Let $J \subseteq\{1, \ldots, n\}$. Then $J$ is said to be essential for $\mathbf{P}$ when the following conditions hold:

(1) $J \subseteq \operatorname{supp}(\mathbf{P})$;

(2) $\operatorname{dim}\left(\sum_{j \in J} P_{j}\right)=|J|-1$;

(3) for all nonempty proper subset $J^{\prime} \subset J$ we have $\operatorname{dim}\left(\sum_{j \in J^{\prime}} P_{j}\right) \geqslant\left|J^{\prime}\right|$.

Given a closed subset $P \subseteq \mathbb{R}_{\geqslant 0}^{n}$ and a vector $w \in \mathbb{R}^{n}$, then we define $\ell(w, P)=\min \{\langle w, k\rangle$ : $k \in P\}$, where $\langle$,$\rangle stands for the standard inner product in \mathbb{R}^{n}$. If $\ell(w, P)>-\infty$, then we denote by $P^{w}$, or by $\Delta(w, P)$, the subset of $P$ formed by those $k \in P$ such that $\langle w, k\rangle=$ $\ell(w, P)$. The sets of the form $\Delta(w, P)$, for some $w \in \mathbb{R}^{n}$, are called faces of $P$. If $\mathbf{P}=$ $\left(P_{1}, \ldots, P_{n}\right)$ is an $n$-tuple of lattice polytopes contained in $\mathbb{R}_{\geqslant 0}^{n}$, then we denote the $n$-tuple $\left(P_{1}^{w}, \ldots, P_{n}^{w}\right)$ by $\mathbf{P}^{w}$.

Next we state a particular case of [21, Theorem 7] that we need for our purposes (we remark that the mentioned theorem is stated for polynomials with coefficients in any algebraically closed field). Given two $n$-tuples of polytopes $\mathbf{P}$ and $\mathbf{Q}$ of $\mathbb{R}^{n}$ such that $\mathbf{P} \subseteq \mathbf{Q}$, the next result gives a purely combinatorial characterization of when $\mathbf{P}$ counts $\mathbf{Q}$.

Theorem 3.4. [21, p. 127] Let $\mathbf{P}$ and $\mathbf{Q}$ be n-tuples of lattice polytopes contained in $\mathbb{R}_{\geqslant 0}^{n}$ such that $\mathbf{P} \subseteq \mathbf{Q}$. Let us suppose that $\mathbf{Q}$ is nice and cornered and $M\left(\mathbf{Q}^{0}\right)>0$. Then $\mathbf{P}$ counts $\mathbf{Q}$ if and only if $\operatorname{supp}\left(\mathbf{P} \cap \mathbf{Q}^{w}\right)$ contains an essential subset for $\mathbf{Q}^{w}$, for all $w \in \mathbb{R}^{n} \backslash \mathbb{R}_{\geqslant 0}$.

If $I$ is a monomial ideal of $\mathcal{O}_{n}$ of finite colength then we define

$$
a_{i}(I)=\max \left\{\operatorname{ord}\left(I^{\mathrm{L}}\right): \mathrm{L} \subseteq\{1, \ldots, n\},|\mathrm{L}|=n-i+1\right\}
$$

for any $i \in\{1, \ldots, n\}$. Let us observe that $a_{1}(I) \leqslant \cdots \leqslant a_{n}(I)$. The us remark that the definition of $a_{i}(I)$ only depends on $\Gamma_{+}(I)$. Therefore $a_{i}(I)=a_{i}(\bar{I})$, for all $i=1, \ldots, n$. We also define the vector $\mathbf{a}(I)=\left(a_{1}(I), \ldots, a_{n}(I)\right)$. For instance, if $I=\left\langle x y z, x^{a}, y^{b}, z^{c}\right\rangle \subseteq \mathcal{O}_{3}$, where $3<a \leqslant b \leqslant c$, then $\mathbf{a}(I)=(3, b, c)$.

We recall that $a_{i}(I)=\mathcal{L}_{0}^{(i)}(I)$, for all $i=1, \ldots, n$, by Theorem 2.3 , however this equality is not used in the main result.

If $k \in \mathbb{R}^{n}$ then we denote by $|k|$ the sum of the coordinates of $k$.

Theorem 3.5. Let I be a monomial ideal of finite colength of $\mathcal{O}_{n}$. Then

$$
e(I) \leqslant a_{1}(I) \cdots a_{n}(I)
$$

and equality holds if and only if there exist polynomials $g_{1}, \ldots, g_{n} \in \mathbb{C}\left[x_{1}, \ldots, x_{n}\right]$ such that $g_{i}$ is homogeneous of degree $a_{i}(I)$, for all $i=1, \ldots, n$, and $\bar{I}=\overline{\left\langle g_{1}, \ldots, g_{n}\right\rangle}$.

Proof. Since $e(I)=e(\bar{I})$ and $a_{i}(I)=a_{i}(\bar{I})$, for all $i=1, \ldots, n$, we can assume that $I$ is integrally closed. Then $I=\left\langle x^{k}: k \in \Gamma_{+}(I)\right\rangle$ (see for instance [14, Proposition 1.4.6]). Let 
$a_{i}=a_{i}(I)$, for all $i=1, \ldots, n$. Let us denote by $D_{i}$ the convex hull in $\mathbb{R}^{n}$ of the set

$$
\left\{k \in \operatorname{supp}\left(I^{\mathrm{L}}\right):|k|=a_{i}, \mathrm{~L} \subseteq\{1, \ldots, n\},|\mathrm{L}|=n-i+1\right\},
$$

for all $i=1, \ldots, n$. By the definition of $a_{i}$ we have $a_{i}=\operatorname{ord}\left(I^{\mathrm{L}}\right)$, for some $\mathrm{L} \subseteq\{1, \ldots, n\}$ such that $|\mathrm{L}|=n-i+1$, for all $i=1, \ldots, n$. In particular $D_{i} \neq \emptyset$, for all $i=1, \ldots, n$. Let D denote the $n$-tuple of polytopes $\left(D_{1}, \ldots, D_{n}\right)$.

If $\alpha \in \mathbb{R}_{\geqslant 0}$, let $\Delta(\alpha)$ denote the convex hull in $\mathbb{R}^{n}$ of the set $\left\{k \in \mathbb{Z}_{\geqslant 0}^{n}:|k|=\alpha\right\}$ and let $\boldsymbol{\Delta}$ denote the $n$-tuple of polytopes $\left(\Delta\left(a_{1}\right), \ldots, \Delta\left(a_{n}\right)\right)$. It is clear that $\Delta$ is nice and cornered and $M\left(\boldsymbol{\Delta}^{0}\right)=a_{1} \cdots a_{n}>0$. Clearly we have $\mathbf{D} \subseteq \boldsymbol{\Delta}$. We claim that $\mathbf{D}$ counts $\boldsymbol{\Delta}$. To see this we will apply Theorem 3.4.

Let us fix a vector $w=\left(w_{1}, \ldots, w_{n}\right) \in \mathbb{R}^{n} \backslash \mathbb{R}_{\geqslant 0}^{n}$ and let $w_{0}=\min \left\{w_{1}, \ldots, w_{n}\right\}$. Let $\mathrm{L}_{w}$ denote the set of indices $\left\{i: w_{i}=w_{0}\right\}$. Then it is immediate to see that $\ell\left(w, \Delta\left(a_{j}\right)\right)=a_{j} w_{0}$ and $\Delta\left(a_{j}\right)^{w}=\Delta\left(a_{j}\right) \cap \mathbb{R}_{\mathrm{L}_{w}}^{n}$, for all $w \in \mathbb{R}^{n} \backslash \mathbb{R}_{\geqslant 0}^{n}$ and all $j=1, \ldots, n$. Then $\boldsymbol{\Delta}^{w}=\boldsymbol{\Delta}^{\mathrm{L}_{w}}$, for all $w \in \mathbb{R}^{n} \backslash \mathbb{R}_{\geqslant 0}^{n}$. In particular we have the equality

$$
\left\{\boldsymbol{\Delta}^{w}: w \in \mathbb{R}^{n} \backslash \mathbb{R}_{\geqslant 0}^{n}\right\}=\left\{\boldsymbol{\Delta}^{\mathrm{L}}: \mathrm{L} \subseteq\{1, \ldots, n\}, \mathrm{L} \neq \emptyset\right\} .
$$

Let us fix a subset $\mathrm{L} \subseteq\{1, \ldots, n\}, \mathrm{L} \neq \emptyset$. Let $\alpha=|\mathrm{L}|$ and let us consider the set of indices $J_{\mathrm{L}}=\{n+1-\alpha, \ldots, n\}$. Let us show that $J_{\mathrm{L}} \subseteq \operatorname{supp}\left(\mathbf{D} \cap \boldsymbol{\Delta}^{\mathrm{L}}\right)$ and $J_{\mathrm{L}}$ is an essential set for $\Delta^{\mathrm{L}}$.

If $i \in J_{\mathrm{L}}$, then $\alpha \geqslant n-i+1$ and thus $\operatorname{ord}\left(I^{\mathrm{L}}\right) \leqslant \operatorname{ord}\left(I^{\mathrm{L}^{\prime}}\right) \leqslant a_{i}$, for all $\mathrm{L}^{\prime} \subseteq \mathrm{L}$ such that $\left|\mathrm{L}^{\prime}\right|=n-i+1$. In particular, if $\mathrm{L}^{\prime} \subseteq \mathrm{L}$ is any subset such that $\left|\mathrm{L}^{\prime}\right|=n-i+1$, there exists some $k \in \operatorname{supp}\left(I^{\mathrm{L}^{\prime}}\right) \subseteq \operatorname{supp}\left(I^{\mathrm{L}}\right)$ such that $|k|=a_{i}$. Then $D_{i} \cap \Delta\left(a_{i}\right)^{\mathrm{L}} \neq \emptyset$, for all $i \in J_{\mathrm{L}}$. That is, we have $J_{\mathrm{L}} \subseteq \operatorname{supp}\left(\mathbf{D} \cap \Delta^{\mathrm{L}}\right)$. We observe that $\operatorname{dim} \Delta(a)^{\mathrm{L}}=|\mathrm{L}|-1$, for all $a \in \mathbb{R}_{\geqslant 0}$. Moreover it is immediate to see that $\sum_{j \in J_{\mathrm{L}}} \Delta\left(a_{j}\right)^{\mathrm{L}}=\left(\Delta\left(\sum_{j \in J_{\mathrm{L}}} a_{j}\right)\right)^{\mathrm{L}}$. In particular we have $\operatorname{dim} \sum_{j \in J_{\mathrm{L}}} \Delta\left(a_{j}\right)^{\mathrm{L}}=|\mathrm{L}|-1$. Then we observe that $J_{\mathrm{L}}$ verifies immediately conditions (2) and (3) of the definition of essential subset for $\Delta^{\mathrm{L}}$ (see Definition 3.3). Thus we deduce that D counts $\boldsymbol{\Delta}$, by Theorem 3.4.

In particular, there exist homogeneous polynomials $g_{i} \in \mathbb{C}\left[D_{i}\right], i=1, \ldots, n$, such that the map $G=\left(g_{1}, \ldots, g_{n}\right): \mathbb{C}^{n} \rightarrow \mathbb{C}^{n}$ verifies that $G^{-1}(0)$ is finite and $m(G)=M\left(\boldsymbol{\Delta}^{0}\right)=$ $a_{1} \cdots a_{n}$. Since $g_{i}$ is homogeneous, for all $i=1, \ldots, n$, and $G^{-1}(0)$ is finite, we conclude that $G^{-1}(0)=\{0\}$. Let $I(G)$ be the ideal of $\mathcal{O}_{n}$ generated by $g_{1}, \ldots, g_{n}$. Then $I(G)$ has finite colength and $e(I(G))=a_{1} \cdots a_{n}$. We assume that $I$ is monomial and integrally closed, then $I(G) \subseteq I$. This implies $a_{1} \cdots a_{n}=e(I(G)) \geqslant e(I)$.

Then by the Rees' multiplicity theorem (see for instance [11, p. 147] or [14, p. 222]), the equality $e(I(G))=e(I)$ holds if and only if $I=\bar{I}=\overline{\left\langle g_{1}, \ldots, g_{n}\right\rangle}$.

Let $G$ denote a homogeneous polynomial map $\mathbb{C}^{n} \rightarrow \mathbb{C}^{n}$ such that $G^{-1}(0)=\{0\}$. Let us denote by $I(G)$ the ideal of $\mathcal{O}_{n}$ generated by the component functions of $G$. We remark that the integral closure of $I(G)$ is not always a monomial ideal, as is shown by the map $G:\left(\mathbb{C}^{2}, 0\right) \rightarrow\left(\mathbb{C}^{2}, 0\right)$ given by $g(x, y)=\left(x y+x^{2}, y^{3}\right)$. 
Let $I$ be a monomial ideal of $\mathcal{O}_{n}$ of finite colength and let $\mathbf{v}=(1, \ldots, 1) \in \mathbb{R}^{n}$. Then we denote the face $\Delta\left(\mathbf{v}, \Gamma_{+}(I)\right)$ by $\Delta_{0}(I)$. Let us observe that the elements $k \in \Gamma_{+}(I)$ such that $|k|=\operatorname{ord}(I)$ are contained in $\Delta_{0}(I)$.

Remark 3.6. Let $I$ be a monomial ideal of $\mathcal{O}_{n}$ of finite colength satisfying the condition $e(I)=a_{1} \cdots a_{n}$, where $a_{i}=a_{i}(I), i=1, \ldots, n$. Then, as we have seen in the proof of Theorem 3.5, the ideal $\bar{I}$ admits a reduction $\left\langle g_{1}, \ldots, g_{n}\right\rangle$, where $g_{i}$ is a homogeneous polynomial of $\mathbb{C}\left[x_{1}, \ldots, x_{n}\right]$ of degree $a_{i}$, for all $i=1, \ldots, n$. Let $d=\operatorname{dim} \Delta_{0}(I)$. Then, as a consequence of [1, Theorem 2.10], where all the reductions of monomial ideals are characterized, it follows that $a_{1}=\cdots=a_{d}=a_{d+1}$. In particular, if $a_{1}<a_{2}$, then $\operatorname{dim} \Delta_{0}(I)=0$, that is, the face $\Delta\left(\mathbf{v}, \Gamma_{+}(I)\right)$ is a vertex.

Let us also observe that, by [1, Theorem 2.10], the condition $e(I)=a_{1} \cdots a_{n}$ forces the face $\Delta_{0}(I)$ to intersect all faces of $\Gamma_{+}(I)$ of dimension $n$. We conjecture that it is possible to obtain a characterization of the condition $e(I)=\prod_{i=1}^{n} a_{i}(I)$ in terms of some property of the tree determined by the vertexes and edges of $\Gamma_{+}(I)$.

Example 3.7. Let us consider the ideal of $\mathcal{O}_{3}$ given by $I=\left\langle x^{a}, y^{b}, z^{c}, x y, x z, y z\right\rangle$, where $2 \leqslant a \leqslant b \leqslant c$. Then $\mathbf{a}(I)=(2,2, c)$. Moreover $e(I)=2+a+b+c$. Then we observe that $e(I) \leqslant 4 c$ and equality holds if and only if $a=b=c=2$.

Here we illustrate Remark 3.6. Let us observe that the face $\Delta_{0}(I)$, contains the convex hull of the supports of the monomials $x y, x z, y z$. Hence $\operatorname{dim} \Delta_{0}(I)=2$. Then $I$ does not satisfy the relation $e(I)=a_{1}(I) a_{2}(I) a_{3}(I)$ if $c>2$, by Remark 3.6.

Example 3.8. Let $I$ be the ideal of $\mathcal{O}_{n}$ generated by $x^{k}, x_{1}^{a_{1}}, \ldots, x_{n}^{a_{n}}$, where $k \in \mathbb{Z}_{\geqslant 0}^{n}$, $k \neq 0$, and $a_{1}, \ldots, a_{n}$ are integers such that $|k| \leqslant a_{1} \leqslant \cdots \leqslant a_{n}$. We recall that $|k|$ denotes the sum of the coordinates of $k$. Then we have $\mathbf{a}(I)=\left(k_{1}+\cdots+k_{n}, a_{2}, \ldots, a_{n}\right)$ and $e(I)=k_{1} a_{2} \cdots a_{n}+\cdots+a_{1} \cdots a_{n-1} k_{n}$. Therefore it is immediate to see that $e(I)=\prod_{i=1}^{n} a_{i}(I)$ if and only if $a_{1}=\cdots=a_{n}$.

Let $\operatorname{lct}(I)$ denote the $\log$ canonical threshold of an ideal $I$ of $\mathcal{O}_{n}$ and let $\mu(I)$ denote the inverse $1 / \operatorname{lct}(I)$, which is also known as the Arnold index of $I$. By a result of de FernexEin-Mustaţă [8, Theorem 1.4], if $I$ is an ideal of $\mathcal{O}_{n}$ of finite colength, then $e(I) \geqslant(n \mu(I))^{n}$ and equality holds if and only if $\bar{I}=\mathbf{m}_{n}^{\text {ord(I) }}$. In particular, using (3), we have

$$
(n \mu(I))^{n} \leqslant e(I) \leqslant \mathcal{L}_{0}^{(1)}(I) \cdots \mathcal{L}_{0}^{(n)}(I) .
$$

We recall that, by a result of Howald [13], if $I$ is a monomial ideal then

$$
\mu(I)=\min \left\{\alpha>0: \alpha \mathbf{v} \in \Gamma_{+}(I)\right\}
$$

where $\mathbf{v}=(1, \ldots, 1) \in \mathbb{R}^{n}$. Let us denote the number on the right hand of (9) by $\alpha(I)$. Then, as a consequence of (7) and [8, Theorem 1.4] we obtain the following conclusion, which is a combinatorial result. 
Corollary 3.9. Let I be a monomial ideal of $\mathcal{O}_{n}$ of finite colength. Then

$$
a_{1}(I) \cdots a_{n}(I) \geqslant(n \alpha(I))^{n}
$$

and equality holds if and only if $\bar{I}=\mathbf{m}_{n}^{\text {ord }(I)}$.

Acknowledgement. Part of this work was developed during the stay of the author at the Max Planck Institute for Mathematics, Bonn (Germany), in April 2011 and the Department of Mathematics of Saitama University (Japan) in March 2012. The author wishes to thank these institutions for their hospitality and financial support.

\section{REFERENCES}

[1] Bivià-Ausina, C. Nondegenerate ideals in formal power series rings, Rocky Mountain J. Math 34, No. 2 (2004), 495-511.

[2] Bivià-Ausina, C. Jacobian ideals and the Newton non-degeneracy condition, Proc. Edinb. Math. Soc. (2) 48, No. 1 (2005), 21-36.

[3] Bivià-Ausina, C. Local Eojasiewicz exponents, Milnor numbers and mixed multiplicities of ideals, Math. Z. 262, No. 2 (2009), 389-409.

[4] Bivià-Ausina, C. Joint reductions of monomial ideals and multiplicity of complex analytic maps, Math. Res. Lett. 15, No. 2 (2008), 389-407.

[5] Bivià-Ausina, C. and Encinas, S. Lojasiewicz exponent of families of ideals, Rees mixed multiplicities and Newton filtrations, Rev. Mat. Complut. 26 (2013), No. 2, 773-798.

[6] Bivià-Ausina, C. and Fukui, T. Mixed Eojasiewicz exponents, log canonical thresholds of ideals and bi-Lipschitz equivalence, preprint, arXiv:1405.2110 [math.AG]

[7] Cox, D., Little, J. and O'Shea, D. Using Algebraic Geometry, 2nd. Edition, Graduate Texts in Mathematics 185. Springer-Verlag (2005).

[8] de Fernex, T., Ein, L. and Mustaţă, M. Multiplicities and log canonical threshold, J. Algebraic Geom. 13 (2004), No. 3, 603-615.

[9] Eisenbud, D. Commutative algebra with a view toward algebraic geometry, Graduate Texts in Mathematics 150, Springer-Verlag (2004).

[10] Ewald, G. Combinatorial convexity and algebraic geometry, Graduate Texts in Mathematics 168. Springer-Verlag (1996).

[11] Herrmann, M., Ikeda, S. and Orbanz, U. Equimultiplicity anb Blowing Up. An Algebraic Study with an Appendix by B. Moonen, Springer, Berlin (1988)

[12] Hickel, M. Fonction asymptotique de Samuel des sections hyperplanes et multiplicité, J. Pure Appl. Algebra 214 (2010), No. 5, 634-645.

[13] Howald, J.A. Multiplier ideals of monomial ideals, Trans. Amer. Math. Soc. 353 (2001), no. 7, 2665-2671.

[14] Huneke, C. and Swanson, I. Integral Closure of Ideals, Rings, and Modules, London Math. Soc. Lecture Note Series 336 (2006), Cambridge University Press.

[15] Lejeune, M. and Teissier, B. Clôture intégrale des idéaux et equisingularité, with an appendix by J.J. Risler. Centre de Mathématiques, École Polytechnique (1974) and Ann. Fac. Sci. Toulouse Math. (6) 17, No. 4 (2008), 781-859.

[16] Li, T.Y. and Wang, X. The BKK root count in $\mathbb{C}^{n}$, Math. Comp. 65, No. 216 (1996), $1477-1484$.

[17] Rees, D. Valuations associated with a local ring II, J. London Math. Soc. 31 (1956), 228-235. 
[18] Rees, D. Lectures on the asymptotic theory of ideals, London Math. Soc. Lecture Note Series 113 (1988), Cambridge University Press.

[19] Rees, D. Generalizations of reductions and mixed multiplicities, J. London Math. Soc. (2) 29 (1984), $397-414$.

[20] Rojas, M. A convex geometric approach to counting the roots of a polynomial system, Theoret. Comput. Sci. 133, No. 1 (1994), 105-140.

[21] Rojas, M. and Wang, X. Counting affine roots of polynomial systems via pointed Newton polytopes, J. Complexity 12, No. 2 (1996), 116-133.

[22] Teissier, B. Cycles évanescents, sections planes et conditions de Whitney, Singularités à Cargèse, Astérisque, no. 7-8 (1973), 285-362.

[23] Teissier, B. Some resonances of Eojasiewicz inequalities, Wiad. Mat. 48, No. 2 (2012), 271-284.

[24] Vasconcelos, W. Integral closure. Rees algebras, multiplicities, algorithms. Springer Monographs in Mathematics. Springer-Verlag, Berlin, 2005.

Institut Universitari de Matemàtica Pura i Aplicada, Universitat Politècnica de València, CAmí de Vera, s/n, 46022 València, Spain

E-mail address: carbivia@mat.upv.es 